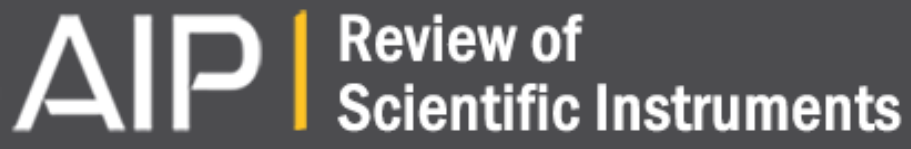

\section{Measurement of total ultrasonic power using thermal expansion and change in buoyancy of an absorbing target}

P. K. Dubey, Yudhisther Kumar, Reeta Gupta, Anshul Jain, and Chandrashekhar Gohiya

Citation: Review of Scientific Instruments 85, 054905 (2014); doi: 10.1063/1.4878625

View online: $\mathrm{http} / / / \mathrm{dx}$.doi.org/10.1063/1.4878625

View Table of Contents: http://scitation.aip.org/content/aip/journal/rsi/85/5?ver=pdfcov

Published by the AIP Publishing

\section{Articles you may be interested in}

Automation of the resistance bridge calibrator

AIP Conf. Proc. 1552, 392 (2013); 10.1063/1.4819572

Impurity and thermal modelling of SPRT fixed-points

AIP Conf. Proc. 1552, 283 (2013); 10.1063/1.4819554

Stray thermal influences in zinc fixed-point cells

AIP Conf. Proc. 1552, 265 (2013); 10.1063/1.4819551

A compact and miniaturized high resolution capacitance dilatometer for measuring thermal expansion and magnetostriction

Rev. Sci. Instrum. 83, 095102 (2012); 10.1063/1.4748864

Precision and Accuracy in Viscometric Measurements on Foods

J. Rheol. 27, 621 (1983); 10.1122/1.549731

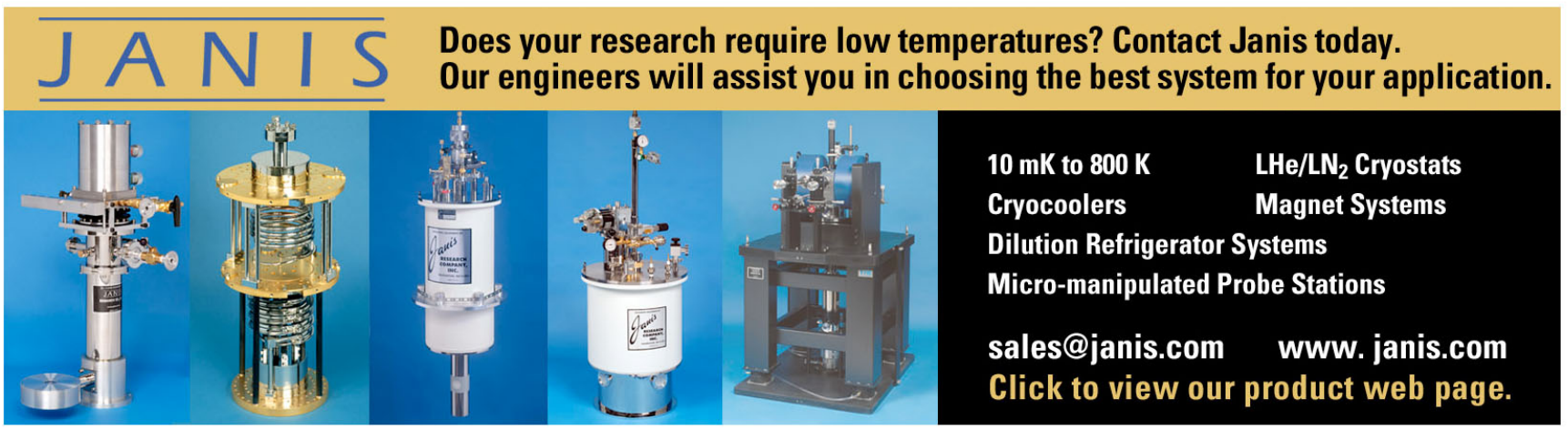




\title{
Measurement of total ultrasonic power using thermal expansion and change in buoyancy of an absorbing target
}

\author{
P. K. Dubey, ${ }^{\text {a) }}$ Yudhisther Kumar, Reeta Gupta, Anshul Jain, and Chandrashekhar Gohiya \\ Acoustics, Ultrasonics, Vibration Standards and Electronics Instrumentation Cell, CSIR-National Physical \\ Laboratory, New Delhi 110012, India
}

(Received 23 February 2014; accepted 6 May 2014; published online 28 May 2014)

\begin{abstract}
The Radiation Force Balance (RFB) technique is well established and most widely used for the measurement of total ultrasonic power radiated by ultrasonic transducer. The technique is used as a primary standard for calibration of ultrasonic transducers with relatively fair uncertainty in the low power (below $1 \mathrm{~W}$ ) regime. In this technique, uncertainty comparatively increases in the range of few watts wherein the effects such as thermal heating of the target, cavitations, and acoustic streaming dominate. In addition, error in the measurement of ultrasonic power is also caused due to movement of absorber at relatively high radiated force which occurs at high power level. In this article a new technique is proposed which does not measure the balance output during transducer energized state as done in RFB. It utilizes the change in buoyancy of the absorbing target due to local thermal heating. The linear thermal expansion of the target changes the apparent mass in water due to buoyancy change. This forms the basis for the measurement of ultrasonic power particularly in watts range. The proposed method comparatively reduces uncertainty caused by various ultrasonic effects that occur at high power such as overshoot due to momentum of target at higher radiated force. The functionality of the technique has been tested and compared with the existing internationally recommended RFB technique. (C) 2014 AIP Publishing LLC. [http://dx.doi.org/10.1063/1.4878625]
\end{abstract}

\section{INTRODUCTION}

The application of ultrasound has been enormously increased. ${ }^{1}$ In industries non-destructive testing and evaluation (NDT and E), ultrasonic cleaning, plastic soldering, and sonochemistry have great importance. There are various medical applications of ultrasound such as medical imaging (echocardiography) which mostly concern soft tissues. ${ }^{2-5}$ Thus, it is of the great importance, either for the safety of the patients or for the understanding of the experimental results, the knowledge of the acoustic field output is necessary. 6,7

The power generated from ultrasonic transducer ranges from few milliwatts for diagnostic equipments to several watts for physiotherapy applications. Absolute measurement techniques provide accurate reference information and the results of measurements are capable of being monitored over both short and long periods, forming the basis of objective in national and international comparison and assessment. ${ }^{4,8}$ The time average ultrasonic power emitted by the source transducer is one of the key values taken into account. ${ }^{9}$ According to both fundamental International Electrotechnical Commission (IEC) standards dealing with acoustic output of medical ultrasonic devices, that of diagnostic devices (IEC 61157) and that of therapeutic devices (IEC 61689), the ultrasonic power is to be measured and declared. ${ }^{10,11}$

The technique recommended in IEC 61161 uses a radiation force balance (RFB). ${ }^{6}$ An appropriately constructed target, properly aligned in a steady state under ultrasound field, is subjected to a radiation force given by ${ }^{12-14}$

$$
F=W / c(T),
$$

${ }^{a)}$ Electronic mail: premkdubey@gmail.com. where $W$ is the time averaged spatially integrated power intercepted by the target and $c$ is the propagation velocity of ultrasound in the medium used as a function of temperature. ${ }^{15-17}$ Time averaging of ultrasound occurs because under practical circumstances, the inertia of the target causes it to effectively integrate pulses into a corresponding steady state force. Spatial integration is exploited by using targets larger in cross section than the incident beam, ${ }^{13}$

$$
W=\Delta m g c(T),
$$

where $\Delta m$ is the apparent change in mass caused by the radiation force and $g$ is the value of gravity at the experimental site. The electro-acoustic radiation conductance of the ultrasonic transducer is given by ${ }^{18,19}$

$$
G=W / V_{r m s}^{2},
$$

where $V_{\mathrm{rms}}$ is the root mean square voltage applied to the transducer. The radiation conductance of ultrasonic transducer is the characteristic parameter and remains constant at a given frequency within the linear range of the ultrasonic output. The manufacturer uses the value of $G$ to produce the desired acoustic output at the specified frequency (generally at resonance) by feeding the desired voltage input. ${ }^{19}$

CSIR National Physical Laboratory, India (NPLI) maintains primary standard for calibration of ultrasonic transducers using radiation force balance method as per IEC 61161. The change in weight is measured using highly sensitive microbalance (Mettler Toledo-XP 56). ${ }^{20}$

Specially designed polyurethane rubber (PUR) material based two layered absorber (HAM-A) procured from Precision Acoustics ${ }^{21}$ is used as an absorbing target in the system. The weight of the target without ultrasound is measured 
and then excited at the desired frequency to generate ultrasound. Due to radiation force exerted on the target the apparent change in weight is measured with a highly sensitive microbalance. Time averaged specially integrated total ultrasonic power is calculated using Eq. (2). The method works extremely perfect for the measurement of ultrasonic power below $1 \mathrm{~W}$.

Particularly in few watts region due to high radiated force the overshoot in the balance output occurs due to increased momentum of the target. This introduces error in the estimation ultrasonic power. If the target is slightly misaligned (not exactly perpendicular to ultrasonic beam) slight horizontal movement, which may be even difficult to see, introduces extra error. If sufficient time is allowed to stabilize, additional error due to local thermal heating of the target takes place. In addition cavitations and acoustic streaming dominate, which causes increase in overall uncertainty in the estimation of ultrasonic power in few watts region. ${ }^{6,18}$ However, literature specifies that ultrasonic absorber (particularly HAM-A) can be used up to $20 \mathrm{~W}$ of ultrasonic power subject to limit the ultrasonic exposure time less than or equal to $10 \mathrm{~s} .{ }^{21}$

We propose a new technique which uses the microbalance outputs before energizing the transducer and just after excitation is over (source switched off). The method utilizes linear thermal expansion of absorber to measure total ultrasonic power more suitable at high power level. The ultrasonic energy radiated by the ultrasonic transducer for a specified duration of time increases the local (exposed area) temperature of the absorbing target thereby increasing its volume. The increase in volume of the target due to thermal expansion can be precisely monitored by measuring the apparent change in mass with the help of a highly sensitive balance. The apparent change in mass is related to the heat generated in the target which is proportional to the total ultrasonic power absorbed by it. The functionality of the approach has been tested and verified with conventional primary calibration standard of RFB method. A common error that is observed in conventional technique is overshoot in the mass measurement at higher force level due to momentum of the target. Other source is movement of target due to sudden application of large force causes variations in the mass value measurement. The proposed technique is less sensitive to these errors caused by transient disturbances in apparent mass measurement due to radiation force of higher level.

\section{AUTOMATED RADIATION FORCE BALANCE SETUP AT NPLI}

Recently, the RFB of NPLI has been upgraded and automated. The improved system records the dynamic variation in the output of microbalance. The voltage applied to the transducer is controlled by a closed loop system. The closed loop system utilizes either Digital Storage Oscilloscope (DSO Tektronix: TDS210) or DC converter module for voltage control. ${ }^{8}$

Fig. 1 shows the block diagram of an automated radiation force balance system. The water bath is made up of Perspex with its inner length, breadth, and height as $300 \mathrm{~mm}$, $300 \mathrm{~mm}$, and $170 \mathrm{~mm}$, respectively. The water bath has a removable lid by which the bath is covered after fixing the

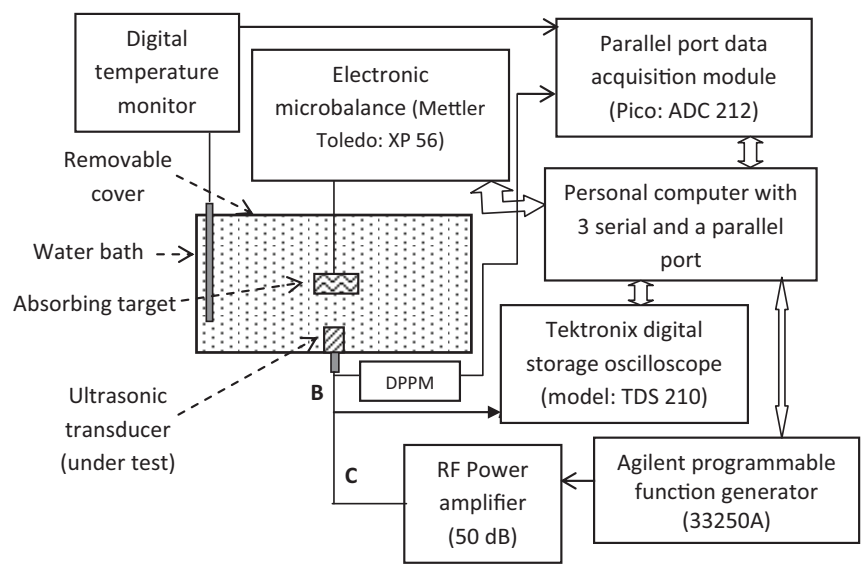

FIG. 1. Block diagram showing the parts of an automated radiation force balance setup at NPL, India used to measure total ultrasonic power of a transducer.

transducer and hanging the absorbing target. The specially designed polyurethane rubber is used as an absorbing target. ${ }^{21}$ The target dimensions are $50 \mathrm{~mm} \times 60 \mathrm{~mm} \times 14 \mathrm{~mm}$. LM 35 sensor based digital temperature monitor designed in the laboratory is used for the measurement of water bath temperature with $\pm 0.2{ }^{\circ} \mathrm{C}$ accuracy. ${ }^{22}$ It also provides amplified (5 times) analog output which is then fed to the computer through pico ADC212 parallel port data acquisition module. The propagation velocity of ultrasound is taken at the measured temperature using standard literature. ${ }^{15,16}$ Highly sensitive dynamic weighing microbalance (Mettler Toledo: XP 56) is used to measure the change in the weight of the absorber. The microbalance is interfaced to the personal computer via RS 232 port for its data acquisition.

Transducer is excited at the desired voltage and frequency using Agilent arbitrary waveform, generator (model: $33250 \mathrm{~A})$, and $\mathrm{E}$ and I fixed gain $(50 \mathrm{~dB})$ power amplifier. A coaxial cable of about $1 \mathrm{~m}$ is used at the output of amplifier to connect the transducer. The input voltage to the transducer is measured and monitored with the help of digital storage oscilloscope. The RF input voltage fed to the transducer is also measured with in-house developed dual peak to peak detector module (DPPM) in order to suppress the errors caused by the connecting coaxial cable to DSO ${ }^{8}$ Control and automation software has been developed in LabVIEW from which different parameters such as RF voltage, frequency, transducer excitation period, and transducer off period, etc., can be set and controlled.

\section{BUOYANCY CHANGE OF TARGET DUE TO THERMAL HEATING}

Due to absorption of ultrasonic waves the temperature of the absorber increases. The apparent weight (force) of the absorber immersed in water is given by

$$
F=\left[m_{a b}-\left(\rho_{w t} \cdot V_{0 a b}\right)\right] \cdot g,
$$

where $m_{a b}$ is the mass of absorber in free space, $\rho_{w t}$ is the density of water, $V_{\text {oab }}$ is the volume of absorber used, and $g$ is the acceleration due to gravity at the experimental site (Fig. 2). 


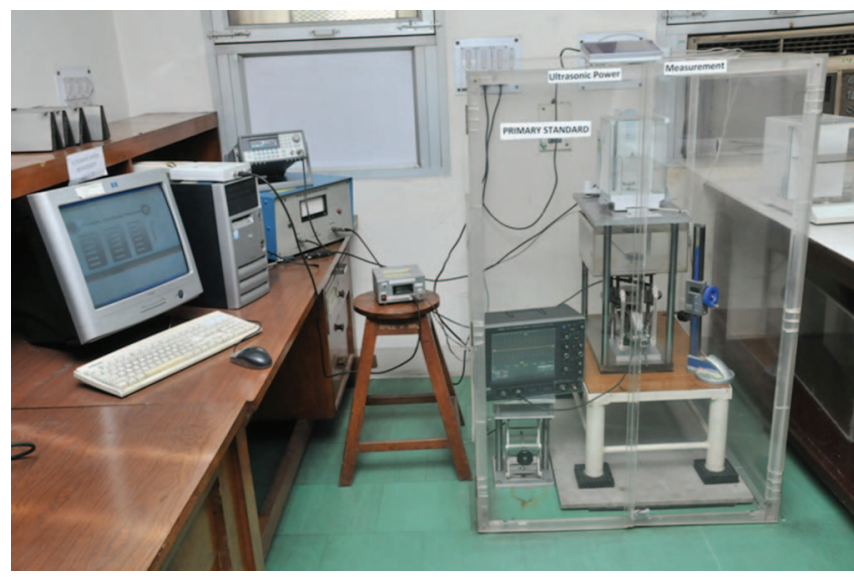

FIG. 2. Snapshot showing the devices used in radiation force balance setup at NPL, India used to measure total ultrasonic power of a transducer.

As the absorber expands due to heating, considering the linear thermal expansion of the absorber Eq. (4) becomes

$$
F=\left[m_{a b}-\left(\rho_{w t} . V_{o a b}\right)\left(1+3 \alpha_{l a b} \Delta T_{a b}\right)\right] . g,
$$

where $\alpha_{l a b}$ is the coefficient of linear thermal expansion of absorber and $\Delta T$ is the change in the localized temperature of the absorber. The apparent change in absorber mass submerged in water due to linear thermal expansion will be

$$
\Delta m_{T}=3 \rho_{w t} . V_{0 a b} \alpha_{l a b} \Delta T_{a b} .
$$

The absorbing target is attached with aluminium disc (diameter $37.7 \mathrm{~mm}$ and $5 \mathrm{~mm}$ thickness) to provide support and helps in hanging the target as shown in Fig. 3. The aluminium disc also increases the total apparent mass of target in water which reduces the target movement at higher radiation force level. As the volume of aluminium disc is small in comparison with the absorber and is in direct contact with the absorber, the raise in the temperature of the absorber will also raise the temperature of the aluminium disc. Considering the thermal expansion of aluminium also in target, Eq. (6) can be

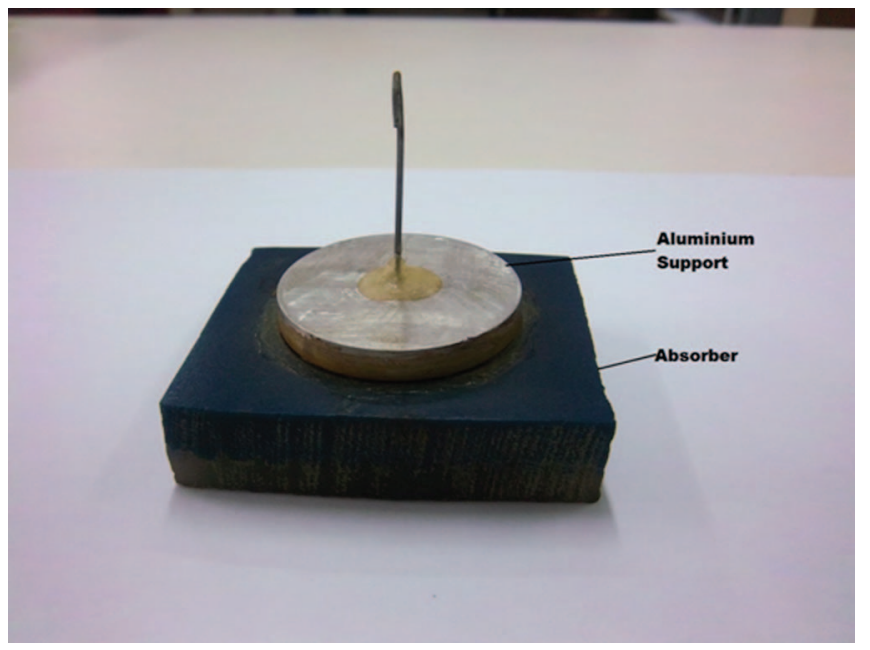

FIG. 3. Snap of the target showing absorber attached to aluminium disc with an arrangement to hang it to microbalance. modified as

$$
\Delta T_{t \arg e t}=\frac{\Delta m_{T}}{3 \rho_{w t} .\left(V_{0 a b} \alpha_{l a b}+V_{0 a l} \alpha_{l a l}\right)} .
$$

The initial volumes of absorber $\left(V_{0 a b}\right)$ and aluminium disc $\left(\alpha_{\text {lal }}\right)$ are known. The values of coefficient of linear thermal expansion of absorber $\left(\alpha_{l a b}\right)$ and aluminium $\left(\alpha_{l a l}\right)$ are taken from the literature. ${ }^{16,21,23}$ By measuring the change in mass $\left(\Delta m_{T}\right)$ due to buoyancy and thermal heating of the submerged target and substituting the appropriate values of the denominator, change in temperature of the target $\left(\Delta T_{t \arg e t}\right)$ can be easily obtained.

\section{ESTIMATION OF ULTRASONIC POWER DUE TO LINEAR THERMAL EXPANSION OF ABSORBING TARGET}

The volume of target (absorber $42 \mathrm{~cm}^{3}$ and aluminium disc $\left.5.57 \mathrm{~cm}^{3}\right)$ is known. The change in apparent mass $(\Delta m)$ of target is continuously monitored and recorded. Substituting the literature values of $\alpha_{l a b}(200 \mathrm{ppm}), \alpha_{l a l}(23.1 \mathrm{ppm})$, and density of water $\rho_{w t}$ at the experimental temperature (0.997 $\mathrm{g} / \mathrm{cm}^{3}$ ), then Eq. (7) becomes

$$
\Delta T_{t \text { arg } e t}=\Delta m_{T} / 0.02551 .
$$

Water has higher specific heat capacity compared with the HAM-A target material and also the volume of water used is comparatively large (300 times approximately). Further, according to IEC61161 the width of absorber must be at least double of the diameter of transducer (device under test). Hence, it can be assumed that the ultrasonic power absorbed by the target rises its temperature keeping the water temperature comparatively stable.

Now, the amount of heat, $q_{a b}$, acquired by absorber to raise the temperature of target by $\Delta T$ :

$$
q_{a b}=m C_{a b} \Delta T_{t \text { arg } e t},
$$

where $\mathrm{m}$ is the mass of the absorber and $\mathrm{C}_{\mathrm{ab}}$ is the heat capacity $^{24}$ of the absorbing material. By precisely controlling and measuring the time for which the device is excited ultrasonic power $W$ will be

$$
W=\frac{q_{a b}}{\Delta t} .
$$

\section{RESULT AND DISCUSSION}

Fig. 4 shows the typical variation in microbalance output with time at different excitation voltages of $10 \mathrm{MHz}, 0.75$ in. transducer (Panametrics NDT, model V315). It is clear from the figure that when the transducer is energized for relatively lower ultrasonic power level, the balance output seems approximately stable. When energized for higher ultrasonic power, gradual decrease in the microbalance output can be noticed; this is clearly visible in $21.7 V_{\text {rms }}$ response. Generally, for the estimation of $\Delta \mathrm{m}$ in conventional radiation force method, balance output is recorded immediately before energizing the transducer and just after it energizes. This gives the 


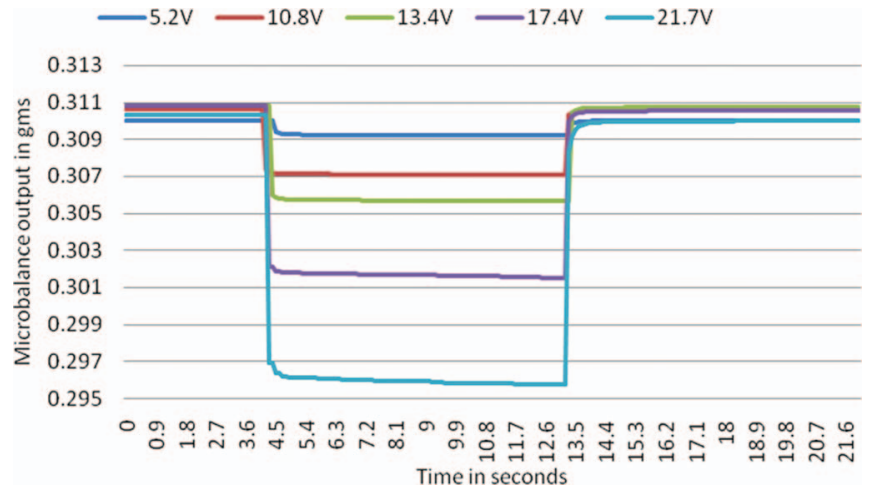

FIG. 4. Variation in the microbalance output with time at different excitation levels of Panametrics $10 \mathrm{MHz}$ transducer.

change in apparent mass of target due to radiation force and the total ultrasonic power is calculated using Eq. (2).

This method is universally adopted and is being used by the National Metrology Institutes (NMIs) as a primary standard for calibration of ultrasonic transducers as per IEC61161. The technique works fine for lower power range. But as we move above in few watts region, the momentum of the target introduces additional source of error. This can be seen in the response of $35 \mathrm{~V}$ and $43 \mathrm{~V}$ excitations in Fig. 5. On recording the output of microbalance just after excitation in the overshoot region, it will result in overestimation of ultrasonic power than the actual, due to momentum. Producing delay in considering the mass value at the overshoot may result in further error (increased $\Delta \mathrm{m}$ ) due to thermal heating of the absorbing target. As the target heats up due to local rise in the temperature thermal expansion takes place. The continuous decrease in the apparent mass due to thermal expansion is depicted in Fig. 5 and relatively high at $43 \mathrm{~V}$ level.

One method of overshoot correction could be an approach of extrapolation and estimation of back points using slope due to thermal expansion. But this approach requires post processing and hence is time consuming. Another method is to consider the raising edge of microbalance output; this gives comparatively lower overshoot and disturbances at the time when transducer is switched off. This method has been adopted and used to compare with the proposed method of measurement of total ultrasonic power using change in buoyancy of target.

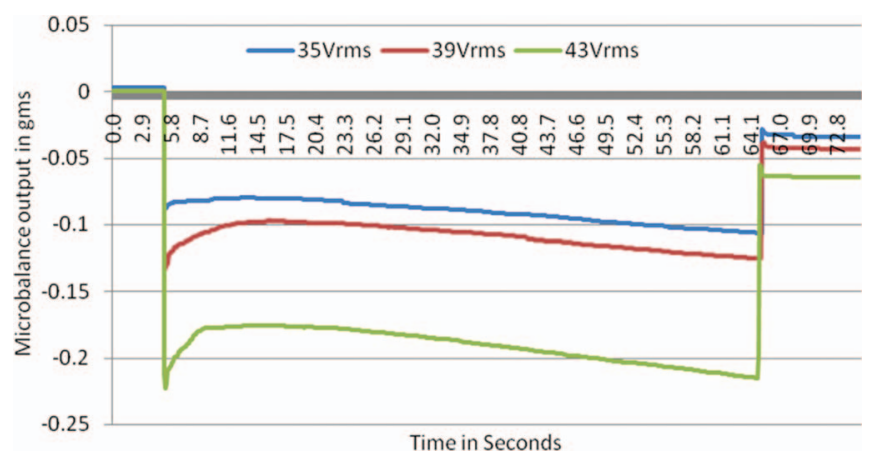

FIG. 5. Deviation in the microbalance output at different excitation levels of the developed transducer of $1.25 \mathrm{MHz}$ used for estimation of total ultrasonic power.

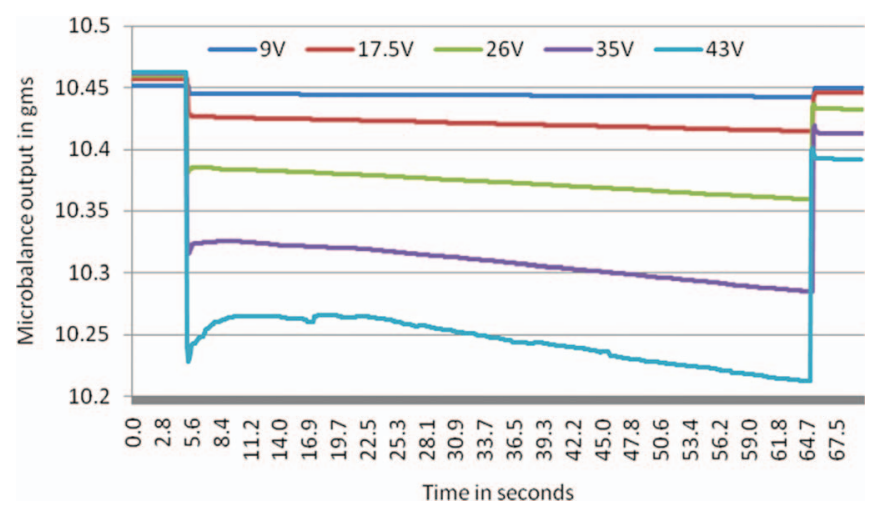

FIG. 6. Variation in the microbalance output with time at different excitation levels of the developed transducer of $1.25 \mathrm{MHz}$.

For the generation of ultrasonic power in watts region a transducer has been developed using gold plated lithium niobate (LiNbO3) crystal with $20 \mathrm{~mm}$ diameter. The transducer is air backed. The developed transducer has been tested for the generation of ultrasonic power up to $5 \mathrm{~W}$ using the radiation force balance facility.

The developed transducer was excited for the duration of $60 \mathrm{~s}( \pm 1 \mathrm{~ms})$ at different excitation levels as shown in Figure 6. From the figure, it is clear that the absorber has been heated at the rates proportional to the ultrasonic power applied. The deviated final value of the balance output indicates the proportional increase in the apparent mass of absorber due to expansion. As the excitation source is switched off the absorber cools down this can be observed in Fig. 7. The absorber temperature decreases exponentially and approaches almost stable value of water in about $30 \mathrm{~min}$. Once this state is reached the system is ready for the next observation.

The developed method has been tested and verified for its functionality by comparing the measured results with the well established primary standard (RFB method) having uncertainty of $\pm 4.5 \%$ at coverage factor of $k=2$ (95\% confidence level). For the measurements the developed transducer was used which is driven at its resonant frequency. The resonant frequency was estimated by driving the transducer at fixed voltage and measuring the ultrasonic power at different frequencies in step of $100 \mathrm{~Hz}$. Maximum power recorded was at $1.2325 \mathrm{MHz}$.

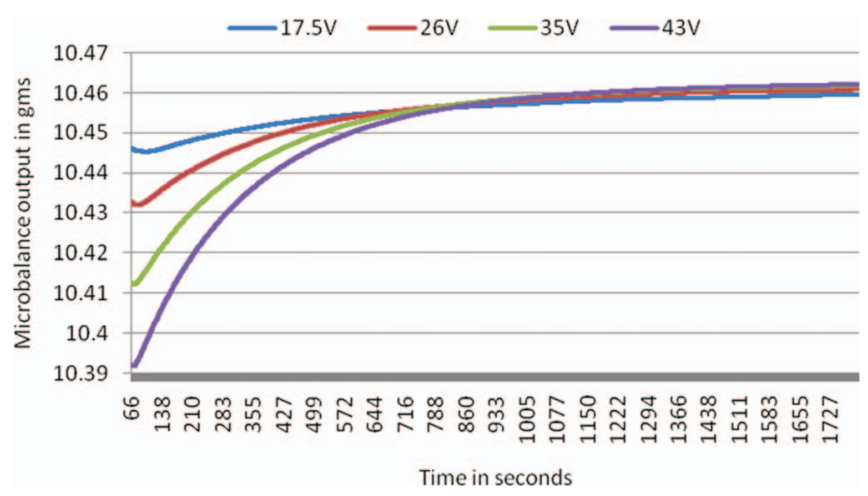

FIG. 7. Typical cooling response of absorbing target from different temperature levels obtained by different excitation voltage levels. 
TABLE I. Comparison of the measurements made by the proposed technique and RFB method on $1.2325 \mathrm{MHz}$, LiNbO3 ultrasonic transducer.

\begin{tabular}{|c|c|c|c|c|c|}
\hline $\begin{array}{l}\text { Input voltage } \\
V_{\mathrm{rms}}\end{array}$ & $\begin{array}{c}\text { Change in mass } \\
\text { due radiation } \\
\text { force }(\mathrm{mg})\end{array}$ & $\begin{array}{l}\text { Change in mass } \\
\text { of target due to } \\
\text { thermal heating (mg) }\end{array}$ & $\begin{array}{c}\text { Ultrasonic power } \\
\mathrm{mW}(\mathrm{W}=\Delta \mathrm{mcg}) \\
( \pm 4.5 \%)\end{array}$ & $\begin{array}{l}\text { Ultrasonic power } \\
(\mathrm{W}=\mathrm{q} / \Delta \mathrm{t}) \mathrm{mW}\end{array}$ & $\%$ deviation \\
\hline 8.8 & 7.161 & 2.575 & 104.59 & 105.94 & 1.27 \\
\hline 13.0 & 16.598 & 6.365 & 243.3 & 261.86 & 7.62 \\
\hline 17.4 & 32.225 & 11.476 & 470.67 & 472.14 & 0.31 \\
\hline 21.7 & 53.198 & 18.516 & 776.99 & 761.77 & 1.95 \\
\hline 26.0 & 70.000 & 24.787 & 1022.40 & 1019.77 & 0.25 \\
\hline 30.4 & 99.790 & 35.131 & 1457.50 & 1445.34 & 0.834 \\
\hline 34.8 & 143.476 & 47.641 & 2095.57 & 1960.01 & 6.46 \\
\hline 39.1 & 182.818 & 61.049 & 2670.18 & 2511.64 & 5.96 \\
\hline 43.4 & 220.815 & 75.109 & 3225.16 & 3090.08 & 4.18 \\
\hline 47.8 & 263.983 & 93.314 & 3855.65 & 3839.06 & 0.43 \\
\hline 52.1 & 303.556 & 106.82 & 4433.65 & 4394.72 & 0.87 \\
\hline
\end{tabular}

In order to verify the functioning of the method for the different time intervals, measurements have been taken for three different time durations $4 \mathrm{~s}, 8 \mathrm{~s}$, and $16 \mathrm{~s}$ at different excitation levels. Each time ultrasonic power was calculated using Eq. (10). The identical voltages were selcted to that of Table I so that the closeness of the measured power level may also be verified. As the three response lines almost overlap each (Fig. 8) other indicating that the output power may be measured even within the small duration of heating period.

Generally the target size is maintained at least double of the transducer diameter and the measurements are carried out in near field zone (close to the transducer). When ultrasound radiated by the transducer is absorbed in the centre of target, then due to local heating in that area thermal expansion takes place which in turn increases the total volume of the absorber. Due to relative rise in temperature at the centre thermal transfer takes place resulting in increase in the temperature of adjacent places. Under such condition the temperature distribution in the absorber will be non-homogeneous. Since, thermal expansion of the absorbing material is highly linear, ${ }^{21}$ the overall increase in volume will be due to total heat absorbed.

If the transducer is switched off, which stops further heating of the target in the centre, the thermal transfer continues till the temperature of the absorber becomes homogeneous. The raised temperature of the absorber transfers heat into water and effective cooling of the target takes place. The volume

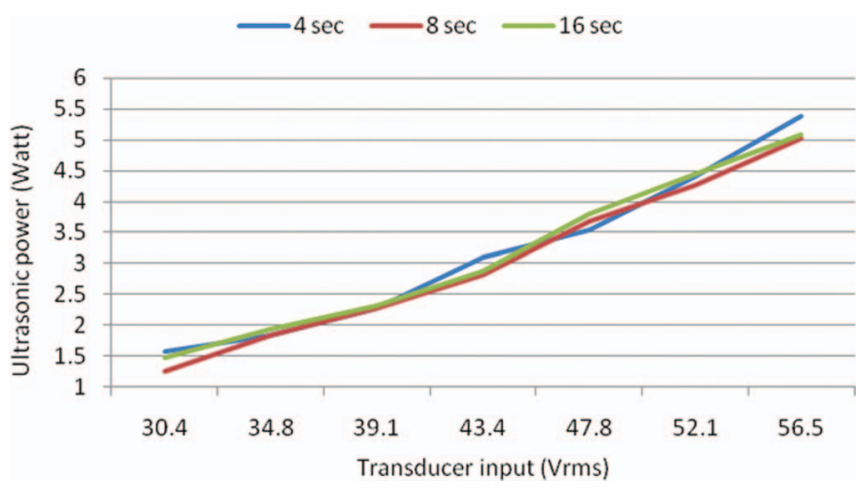

FIG. 8. Estimation of ultrasonic output power of a transducer at different excitation intervals. of water is very high as compared to that of target. Also, due to high specific heat capacity of water and high volume, the amount of heat required would be approximately 1000 times higher to raise its temperature equal to the absorber. Hence, the temperature of water is almost maintained at the ambient temperature. In this experimental setup, the temperature of water was measured and monitored closed to the absorber (at $1 \mathrm{~cm}$ distance). The raise in temperature was within $0.2^{\circ} \mathrm{C}$ which has least significant contribution in the variation of water density. Hence, density of water was considered as constant during experimentation.

\section{CONCLUSIONS}

Method for the measurement of total ultrasonic power by utilizing linear thermal expansion and change in buoyancy of absorbing target has been successfully developed and its functionality has been demonstrated. The approach may be useful for the measurement of ultrasonic power in few watts region by precisely measuring the thermal expansion effect within short duration of time. For estimation of low power, particularly in $\mathrm{mW}$ region thermal response for the longer duration excitation may be considered which will produce significant change in the buoyancy of target; necessary for estimation of ultrasonic power.

On the other hand the target requires about $30 \mathrm{~min}$ for cooling and regaining the normal temperature. This limits the measurement speed of this approach. However, if sufficient time for target cooling is not included it will cause error in the estimation of output power. From the literature it has been observed that the linear thermal expansion of the target material (PUR) also depends on temperature (water temperature). ${ }^{24}$ Hence, knowledge of temperature dependence of target material expansion coefficient is necessary or the measurement may be made at constant temperature of water.

\section{ACKNOWLEDGMENTS}

The authors (particularly P. K. Dubey) would like to thank the Director, CSIR-National Physical Laboratory, for providing the necessary facilities and financial assistance for 
the improvement in ultrasonic power measurement system. Thanks are also due for the financial support provided to one of the authors (Anshul Jain) under CSIR research intern scheme.

${ }^{1}$ M. A. Pleitez, T. Lieblein, A. Bauer, and O. Hertzberg, Rev. Sci. Instrum. 84, 084901 (2013).

${ }^{2}$ G. K. Lewis, Jr. and W. L. Olbricht, Rev. Sci. Instrum. 79, 114302 (2008).

${ }^{3}$ K. Martin, IEEE Trans. Ultrason. Ferroelectr. Freq. Control 35(2), 140-145 (1988).

${ }^{4}$ R. C. Preston and A. J. Livett, IEEE Proc. 131(4), 233-240 (1984).

${ }^{5}$ M. E. Schafer and P. A. Lewin, IEEE Trans. Ultrason. Ferroelectr. Freq. Control 35(2), 102-109 (1988).

${ }^{6}$ IEC Standard 1161 (61161), International Electrotechnical Commission (1998).

${ }^{7}$ N. R. Miller and J. C. Bamber, J. Phys.: Conf. Ser. 1, 128-133 (2004).

${ }^{8}$ P. K. Dubey, A. Kumar, Y. Kumar, R. Gupta, and D. Joshi, Rev. Sci. Instrum. 81, 104904 (2010).

${ }^{9}$ K. Beissner, Metrologia 36, 313-320 (1999).

${ }^{10}$ IEC Standard 1157 (61157), International Electrotechnical Commission (1992).
${ }^{11}$ IEC Standard 1689 (61689), International Electrotechnical Commission (1996).

${ }^{12}$ K. Beissner, J. Acoust. Soc. Am. 76(5), 1505-1511 (1984).

${ }^{13}$ S. E. Fick and F. R. Breckenridge, J. Res. National Inst. Standards and Technol 101, 659-669 (1996).

${ }^{14}$ B. Zeqiri, Output Measurements for Medical Ultrasound, edited by R. C. Preston (Springer-Verlag, 1991), Chap. 3, pp. 41-44.

${ }^{15}$ V. A. Del Grosso and C. A. Mader, J. Acoust. Soc. Am. 52, 1442 (1972)

${ }^{16}$ National Physical laboratory, UK, Kaye and Laby Tables of Physical and Chemical Constants, see www.kayelaby.npl.uk.

${ }^{17}$ S. Rajagopalan, S. J. Sharma, and P. K. Dubey, Rev. Sci. Instrum. 78, 085104 (2007).

${ }^{18}$ T. Kikuchi, S. Sato, and M. Yoshioka, IEEE Trans. Ultrason. Ferroelectr. Freq. Control Conf. (50th Anniversary Conf.), 2197-2200 (2004).

${ }^{19}$ S. E. Fick, J. Res. Nat. Inst. Standards Technol. 113, 281-286 (2008).

${ }^{20}$ Mettler Toledo : XP-56 datasheet, see www.mt.com.

${ }^{21}$ For HAM-A datasheet: Precision Acoustics, see www.acoustics.co.uk.

${ }^{22}$ IC LM35, Temperature sensor datasheet.

${ }^{23}$ C. A. Steevesa, S. L. dos Santos e Lucatob, M. Hea, E. Antinuccia, J. W. Hutchinsonc, and A. G. Evans, J. Mech. Phys. Sol. 55, 1803-1822 (2007).

${ }^{24}$ See www.polyurethanes.basf.de for details of thermal properties of polyurethane rubber. 